\title{
Dredge-up effects in galactic and magellanic planetary nebulae ${ }^{\star}$
}

\author{
R.D.D. Costa ${ }^{1}$, J.A. de Freitas Pacheco ${ }^{2}$, and T.P. Idiart $^{1}$ \\ 1 Instituto Astronômico e Geofísico, Av. Miguel Stéfano 4200, 04301-904 São Paulo, Brazil \\ 2 Observatoire de la Côte d'Azur, BP. 4229, 06304 Nice Cedex 4, France
}

Received February 14; accepted June 29, 2000

\begin{abstract}
Chemical abundances are reported for 23 planetary nebulae in the Small Magellanic Cloud. Average abundances agree with values found for HII regions, suggesting that progenitors of most of these objects have been formed in the last $1-2$ Gyr. The $\mathrm{N} / \mathrm{O}$ vs. $\mathrm{O} / \mathrm{H}$ anticorrelation is confirmed for Magellanic and galactic planetaries, and a more robust result is obtained if objects of similar ages (or masses) are grouped together. For a given class, such a negative correlation suggests that the surface enrichment due to dredge-up episodes is more efficient at lower metallicities, in agreement with recent computations.
\end{abstract}

Key words: galaxies: Magellanic Clouds, abundances Planetary Nebulae

\section{Introduction}

Planetary Nebulae (PN) are the final evolutionary stage of intermediate mass stars, and their chemical abundances have been extensively used to probe the chemical evolution of the Galaxy and the Magellanic Clouds. In these objects, abundances of elements like $\mathrm{He}, \mathrm{C}$ and $\mathrm{N}$ were probably modified at the surface of the progenitor, due to dredge-up episodes prior ejection of the outer envelope. On the other hand, abundances of Ne, S and Ar represent the conditions of the interstellar medium at the epoch of the formation of the parent star.

Oxygen deserves a special attention since it is one of the key elements used to trace the chemical evolution of stellar systems. However, concerning changes in the oxygen abundance along the stellar evolution of the progenitor, we don't have a satisfactory answer yet. If only

Send offprint requests to: R.D.D. Costa

* Based on observations made at European Southern Observatory (Chile) and Laboratório Nacional de Astrofísica (Brazil).

Correspondence to: e-mail: roberto@orion.iagusp.usp.br
CN-cycle products are dredge-up, oxygen remains unchanged, but some reduction is expected if the ON-cycle is operative. Theoretical estimates predict a maximum (uncertain) reduction of about 0.11 dex in the surface abundances of massive progenitors (Renzini \& Voli 1981). The analyses of the $\mathrm{C} / \mathrm{N}$ and $\mathrm{C} / \mathrm{O}$ ratios in galactic supergiants suggest that these stars have dredged-up essentially CN-cycle products and that oxygen remained unchanged (Luck \& Lambert 1985). Concerning PN, different data sets (Köppen et al. 1991; Kingsburgh \& Barlow 1994; Costa \& de Freitas Pacheco 1996) indicate that the oxygen abundances of massive and young planetaries (type I objects) agree with abundances derived from HII regions, consistent with the conclusion derived from galactic supergiants, namely, oxygen was not affected. These first considerations may give some support to the scenario in which oxygen would not be altered by mixing episodes and its abundance reflects the pristine chemical composition of the medium from where PN have been formed out.

Abundance studies performed in the early eighties by Torres-Peimbert (1984) seem to suggest an anticorrelation between the $\mathrm{N} / \mathrm{O}$ ratio and the oxygen abundance. This anti-correlation would be restricted primarily to type I planetaries, since non-type I objects would show a positive correlation, according to other investigations (Torres-Peimbert \& Peimbert 1977; Peimbert \& Serrano 1980). With a sample covering a broader abundance range, Kaler et al. (1990) found that also non-type I planetaries have a negative $\mathrm{N} / \mathrm{O}$ vs. $\mathrm{O} / \mathrm{H}$ correlation. These observations were interpreted by Peimbert (1985) as an evidence that most of the nitrogen in type I planetaries is of secondary origin, coming from oxygen rather than carbon. Such an anti-correlation was noted to be present not only in galactic $\mathrm{PN}$ but also in LMC and SMC data (Aller 1983; Henry et al. 1989).

In the past years, an observational program aiming to obtain chemical abundances of southern $\mathrm{PN}$, including also objects in the Magellanic Clouds, has been carried out at the University of S. Paulo. Chemical abundances for 74 galactic PN included in our survey were 
summarized by Costa \& de Freitas Pacheco (1996), and results for $23 \mathrm{PN}$ in the LMC were reported by de Freitas Pacheco et al. (1993a,b). In the present work we report additional abundance data for $23 \mathrm{PN}$ in the SMC as a part of our survey, and then review the problem of the N/O vs. $\mathrm{O} / \mathrm{H}$ correlation. For galactic $\mathrm{PN}$ we use our sample constituted by 74 objects (Costa \& de Freitas Pacheco 1996), covering an oxygen abundance range of about two orders of magnitude $7.08<\varepsilon(\mathrm{O})<9.11$ (where $\varepsilon(\mathrm{X})=\log (\mathrm{X} / \mathrm{H})$ +12 ). For the LMC we used data by de Freitas Pacheco et al. (1993a,b) and for the SMC, the present results, both supplemented by literature data. The plan of the paper is the following: in Sect. 2 we summarize observations, data reduction and physical properties for the $23 \mathrm{PN}$ in the $\mathrm{SMC}$; in Sect. 3 we discuss the $\mathrm{N} / \mathrm{O}$ vs. $\mathrm{O} / \mathrm{H}$ relation for galactic and Magellanic PN, and in Sect. 4 we present our main conclusions.

\section{SMC data}

\subsection{Observations}

Observations were performed using telescopes at ESO $(1.52 \mathrm{~m})$ and at Laboratório Nacional de Astrofísica (LNA), Brazil $(1.60 \mathrm{~m})$. At ESO a Boller \& Chivens Cassegrain spectrograph was used with a Loral/Lesser CCD and grating allowing a reciprocal dispersion of about $2.5 \AA /$ pixel. At LNA, a Boller \& Chivens Cassegrain spectrograph was also used with a SITe CCD but with a smaller dispersion, namely, $4.4 \AA /$ pixel. A long eastwest slit was used in all observations. The log of the observations is given in Table 1 .

Image reduction and analysis were performed using the IRAF package, including the classical procedure to reduce long slit spectra: bias, dark and flat-field corrections, spectral profile extraction, wavelength and flux calibrations. Atmospheric extinction was corrected using mean coefficients for each observatory, and flux calibration was secured by the observation of standard stars (at least three) every night.

Emission line fluxes were calculated assuming Gaussian profiles, and a Gaussian de-blending routine when necessary. A table with dereddened line intensities is available in electronic form at CDS. Adopting a scale in which $I(\mathrm{H} \beta)=100$, typical errors in the intensities are of about $15 \%$ for lines stronger than 10 and of about $30 \%$ for weaker lines. Interstellar reddening was estimated using the Balmer ratio $\mathrm{H} \alpha / \mathrm{H} \beta$, assuming Case B (Osterbrock 1989) and adopting the extinction law by Cardelli et al. (1989). $E(B-V)$ values derived for each nebula are given in Table 2.

\subsection{Physical parameters}

Electron densities were estimated from the $[\mathrm{SII}]$ ratio $\lambda 6716 / \lambda 6731$ and from the $[\mathrm{ArIV}]$ ratio $\lambda 4711 / \lambda 4740$,
Table 1. Log of the observations

\begin{tabular}{|c|c|c|c|c|}
\hline Object & $\alpha_{2000}$ & $\delta_{2000}$ & Date & Observatory \\
\hline SMP 01 & 002405 & -733722 & 1999 Aug. 19 & $\mathrm{ESO}$ \\
\hline SMP 02 & 003239 & -714159 & 1999 Aug. 19 & ESO \\
\hline SMP 03 & 003421 & -731324 & 1999 Aug. 19 & $\mathrm{ESO}$ \\
\hline SMP 04 & 004042 & -751700 & 1999 Aug. 20 & ESO \\
\hline SMP 05 & 004122 & -724517 & 1999 Jul. 18 & LNA \\
\hline SMP 06 & 004128 & $\begin{array}{lll}-73 & 47 & 07\end{array}$ & 1999 Jul. 18 & LNA \\
\hline SMP 07 & 004228 & -732055 & 1999 Aug. 15 & $\mathrm{ESO}$ \\
\hline SMP 08 & 004325 & -723818 & 1999 Aug. 20 & $\mathrm{ESO}$ \\
\hline SMP 09 & 004521 & -732400 & 1000 Dec. 28 & $\mathrm{ESO}$ \\
\hline SMP 10 & 004700 & -724916 & 1999 Jul. 20 & LNA \\
\hline SMP 11 & 004836 & -725800 & 1999 Jul. 20 & LNA \\
\hline SMP 12 & 004921 & -735259 & 1999 Aug. 16 & ESO \\
\hline SMP 13 & 004952 & -734423 & 1999 Dec. 26 & $\mathrm{ESO}$ \\
\hline SMP 14 & 005035 & -734300 & 1999 Dec. 27 & $\mathrm{ESO}$ \\
\hline SMP 16 & 005127 & $\begin{array}{lll}-72 & 26 & 11\end{array}$ & 1999 Dec. 29 & $\mathrm{ESO}$ \\
\hline SMP 17 & 005156 & -712445 & 1999 Aug. 17 & $\mathrm{ESO}$ \\
\hline SMP 18 & 005158 & -732032 & 1999 Aug. 17 & $\mathrm{ESO}$ \\
\hline SMP 19 & 005311 & -724507 & 1999 Aug. 18 & $\mathrm{ESO}$ \\
\hline SMP 21 & 005631 & -722701 & 1999 Dec. 26 & $\mathrm{ESO}$ \\
\hline SMP 22 & 005837 & -713549 & 1999 Aug. 17 & $\mathrm{ESO}$ \\
\hline SMP 23 & 005842 & -725659 & 1999 Aug. 20 & $\mathrm{ESO}$ \\
\hline SMP 25 & 005941 & -713816 & 1999 Dec. 28 & $\mathrm{ESO}$ \\
\hline N 9 & 004337 & -730226 & 1999 Aug. 15 & $\mathrm{ESO}$ \\
\hline
\end{tabular}

when the appropriate lines were available. Electron temperatures were derived from both [OIII] $\lambda 4363 / \lambda 5007$ and [NII] $\lambda 5754 / \lambda 6584$ line ratios. Whenever these temperatures were comparable, we have adopted a mean value, otherwise the $[\mathrm{OIII}]$ temperature was used to estimate abundances of higher ionization potential ions like $\mathrm{O}^{+2}$, $\mathrm{S}^{+2}, \mathrm{Ar}^{+2,+3}, \mathrm{Ne}^{+2}$ and the [NII] temperature of lower potential ions like $\mathrm{O}^{+}, \mathrm{N}^{+}, \mathrm{S}^{+}$. Temperatures and densities adopted for each nebula are also listed in Table 2. A compilation of physical parameters for Magellanic planetaries was prepared by Richer (1993). A comparison between common objects indicates that the average temperature difference between literature and our estimates amounts to $640 \mathrm{~K}$, with a dispersion of $1680 \mathrm{~K}$. Electron densities have a higher difference (of about $60 \%$ on the average). These differences are probably due to artifacts. They can be related to errors in the flux determination of the [SII] lines, frequently weak and with considerable uncertainties in their fluxes. Different origins of the atomic data used to derive densities from the [SII] ratio could also account for this dispersion, however, it should be emphasized that no systematic effects were observed between the different samples.

\subsection{Chemical abundances}

Ionic abundances were calculated for each ion of interest by solving the statistical equilibrium equations for a three-level atom model, including radiative and collisional transitions. Elemental abundances were then derived 
Table 2. Electron temperatures and densities

\begin{tabular}{lccccc}
\hline Object & $E(B-V)$ & $T_{\mathrm{e}}[\mathrm{OIII}](\mathrm{K})$ & $T_{\mathrm{e}}[\mathrm{NII}](\mathrm{K})$ & $N_{\mathrm{e}}[\mathrm{SII}]\left(\mathrm{cm}^{-3}\right)$ & $N_{\mathrm{e}}[\mathrm{ArIV}]\left(\mathrm{cm}^{-3}\right)$ \\
\hline SMP 01 & 0.11 & 11900 & 11800 & $47600:$ & - \\
SMP 02 & 0.11 & 13700 & 11460 & 4090 & 4270 \\
SMP 03 & 0.04 & 13270 & - & - & $21000:$ \\
SMP 04 & 0.00 & 14980 & $12700:$ & - & 4860 \\
SMP 05 & 0.42 & 12400 & 10800 & 4160 & 2300 \\
SMP 06 & 0.23 & 12800 & 9700 & $>3300:$ & $60000:$ \\
SMP 07 & 0.09 & 15660 & $9300:$ & 1140 & - \\
SMP 08 & 0.02 & 11800 & $10200:$ & 700 & 3780 \\
SMP 09 & 0.60 & 13470 & $10000:$ & 770 & 880 \\
SMP 10 & 0.57 & 9800 & - & $860:$ & - \\
SMP 11 & 0.37 & 14000 & $11500:$ & $1800:$ & - \\
SMP 12 & 0.00 & 13800 & $9100:$ & $880:$ & $370:$ \\
SMP 13 & 0.03 & 10800 & - & 10000 & - \\
SMP 14 & 0.03 & 11600 & - & 430 & 500 \\
SMP 16 & 0.02 & 10700 & - & 3700 & - \\
SMP 17 & 0.27 & 11230 & 10300 & 3270 & 400 \\
SMP 18 & 0.17 & $12400:$ & $12100:$ & 4090 & - \\
SMP 19 & 0.28 & $11570:$ & $9780:$ & 1580 & 2900 \\
SMP 21 & 0.16 & 23500 & $23300:$ & 8400 & 11220 \\
SMP 22 & 0.24 & 22400 & 10620 & 1550 & - \\
SMP 23 & 0.00 & 13700 & $11700:$ & - & 1210 \\
SMP 25 & 0.00 & 38700 & - & 3760 & 5390 \\
N 09 & 0.15 & 12700 & 12000 & 170 & - \\
\hline
\end{tabular}

Table 3. Chemical abundances

\begin{tabular}{lccccccc}
\hline Object & He & $\varepsilon(\mathrm{O})$ & $\varepsilon(\mathrm{N})$ & $\varepsilon(\mathrm{Ne})$ & $\varepsilon(\mathrm{S})$ & $\varepsilon(\mathrm{Ar})$ & type \\
\hline SMP 01 & 0.086 & 8.01 & 7.05 & 7.33 & $5.95:$ & 5.73 & - \\
SMP 02 & 0.110 & 8.28 & 7.07 & 7.70 & $6.13:$ & 5.66 & - \\
SMP 03 & 0.103 & 8.18 & 7.04 & 7.41 & 6.62 & 5.48 & - \\
SMP 04 & 0.132 & 7.92 & - & - & - & 5.46 & $\mathrm{I}(?)$ \\
SMP 05 & 0.120 & $8.51:$ & 7.17 & 7.51 & 6.41 & 5.80 & $\mathrm{I}$ \\
SMP 06 & $0.12:$ & 8.36 & 7.34 & - & 6.35 & 5.88 & $\mathrm{I}$ \\
SMP 07 & 0.102 & 8.17 & 7.14 & 7.46 & - & - & - \\
SMP 08 & 0.116 & 8.24 & 6.74 & 7.75 & 6.15 & 5.77 & - \\
SMP 09 & $0.11:$ & 8.48 & 7.08 & - & 6.49 & 6.27 & $\mathrm{I}$ \\
SMP 10 & $0.14:$ & 8.55 & 7.30 & - & 6.67 & 6.48 & $\mathrm{I}$ \\
SMP 11 & 0.100 & 8.37 & 6.39 & 7.90 & 6.26 & 6.08 & - \\
SMP 12 & $0.11:$ & 8.08 & 7.62 & 7.27 & 6.37 & 5.71 & $\mathrm{I}$ \\
SMP 13 & 0.128 & 8.41 & 6.80 & - & - & 5.73 & $\mathrm{I}$ \\
SMP 14 & 0.116 & 8.41 & 7.13 & - & 6.49 & 5.73 & $\mathrm{I}$ \\
SMP 16 & 0.086 & 8.41 & 6.90 & - & 5.90 & 5.86 & - \\
SMP 17 & $0.14:$ & 8.48 & 7.19 & 7.60 & 6.25 & 6.05 & $\mathrm{I}$ \\
SMP 18 & 0.095 & 7.99 & 6.83 & 7.25 & 6.16 & 5.75 & - \\
SMP 19 & 0.115 & 8.47 & 6.82 & - & 6.14 & 5.57 & $\mathrm{I}$ \\
SMP 21 & $0.15:$ & 7.57 & 7.41 & - & 6.31 & 5.53 & $\mathrm{I}(?)$ \\
SMP 22 & 0.152 & 7.59 & 8.26 & 6.97 & 6.10 & 5.48 & $\mathrm{I}$ \\
SMP 23 & 0.098 & 8.05 & - & 7.38 & - & 5.50 & - \\
SMP 25 & 0.105 & 7.01 & 6.93 & - & 5.54 & 5.09 & - \\
N 09 & 0.085 & 8.20 & 6.52 & 7.55 & 6.28 & 5.79 & - \\
\hline
\end{tabular}

through ionization correction factors (icf) adopted to account for unobserved ions of each element. We used the same icf's adopted in our precedent publications (Costa et al. 1996). Resulting abundances are given in Table 3. Typical errors are about 0.2 dex for $\mathrm{O}, \mathrm{N}, \mathrm{Ne}$ and 0.3 dex for $\mathrm{S}$ and Ar. Helium abundances deserve further attention, since collisional corrections by Clegg (1987) may be overestimated up to a factor of two (Peimbert \& Torres-Peimbert 1987). Here we have adopted Clegg's formulae multiplied by an empirical factor equal to 0.6 , since this gives a much better agreement between $\mathrm{He}^{+}$abundances derived from the recombination lines HeI $\lambda 4471,5876$ and 6678 . In the case of helium, when three digits are given, errors are of the order of 0.004 and, when discrepancies between the three HeI lines are greater than 0.02 the mean value is followed by ":".

Previous chemical studies of $\mathrm{PN}$ in the SMC have shown that taking the whole sample, the average oxygen abundance tends to be higher than the mean values found for type I objects only (see, for instance, Leisy \& Dennefeld 1996), a result not confirmed by the present study, as will be seen later. If some non-type I objects are misclassified, this could be a possible explanation. Galactic type I PN, besides the $\mathrm{He}$ and $\mathrm{N}$ excess, are characterized by their bipolar morphology, average distance to galactic plane and peculiar $\langle\Delta V\rangle$ velocity (Maciel \& Dutra 1992). At the distance of the Clouds, morphologies are not presently available and one should wait for future high angular resolution observations, using 
Table 4. Average abundances

\begin{tabular}{lcccc}
\hline Object & $\varepsilon(\mathrm{O})$ & $\varepsilon(\mathrm{Ne})$ & $\varepsilon(\mathrm{S})$ & $\varepsilon(\mathrm{Ar})$ \\
\hline Type I & $8.33 \pm 0.28$ & $7.33 \pm 0.24$ & $6.35 \pm 0.16$ & $5.87 \pm 0.30$ \\
All & $8.22 \pm 0.27$ & $7.47 \pm 0.23$ & $6.28 \pm 0.20$ & $5.76 \pm 0.25$ \\
HII reg. & 8.13 & 7.22 & 6.32 & 5.78 \\
\hline
\end{tabular}

large aperture southern telescopes (VLT, Gemini) now becoming operational. The Clouds and the Galaxy have different dynamical structures, so the kinematical and space distributions are not directly comparable. Thus, for the moment, He and $\mathrm{N}$ abundances are the only classification criteria. As de Freitas Pacheco et al. (1993a) have already emphasized, since the Clouds have lower metallicities, the self-enrichment condition to be applied is not necessarily the same as that first defined by Peimbert \& Torres-Peimbert (1983). Here we adopted a more conservative position. Since our main goal is to investigate the reality of the anti-correlation $\mathrm{N} / \mathrm{O}$ vs. $\mathrm{O} / \mathrm{H}$, we used only He abundance to classify the objects in our sample, assuming that objects with $\mathrm{He} / \mathrm{H}>0.11$ are genuine type I $\mathrm{PN}$ as indicated in the last column of Table 3. This is a more drastic limit than that assumed either by de Freitas Pacheco et al. (1993a) or by Leisy \& Dennefeld (1996), but it gives a higher confidence in our analysis. We will return to this point later.

Average abundances are shown in Table 4, either for type I PN as for all the sample. Objects with uncertain He abundances were excluded when average values were computed for type I planetaries. For comparison, average abundances for HII regions taken from Dennefeld (1989) are also given, excepting for oxygen, taken from the HII region sample by Russel \& Dopita (1990).

Inspection of Table 4 indicates immediately that no significant differences exist between average abundances of type I PN and the ensemble average, although type I seem to have slightly higher values. However we should emphasize that we are still playing with small numbers and a large sample is necessary to draw a more firm conclusion. Nevertheless our mean values are quite consistent with interstellar medium abundances and, in particular oxygen. It is worth mentioning that Hill et al. (1997), from the study of six K-supergiants, obtained a mean oxygen abundance $\varepsilon(\mathrm{O})=8.14 \pm 0.12$, which agrees both with PN and HII region average values. A similar behavior is observed for galactic (de Freitas Pacheco 1993; Costa et al. 1996) and LMC planetaries (de Freitas Pacheco et al. 1993a). Taking at face value, these results may be interpreted in the sense that oxygen was preserved, not being affected by any dredge-up episode.

\section{The $\mathrm{N} / \mathrm{O}$ vs. $\mathrm{O} / \mathrm{H}$ relation}

Previous studies of galactic $\mathrm{PN}$ suggested the existence of an anti-correlation between $\mathrm{N} / \mathrm{O}$ and $\mathrm{O} / \mathrm{H}$ for type I

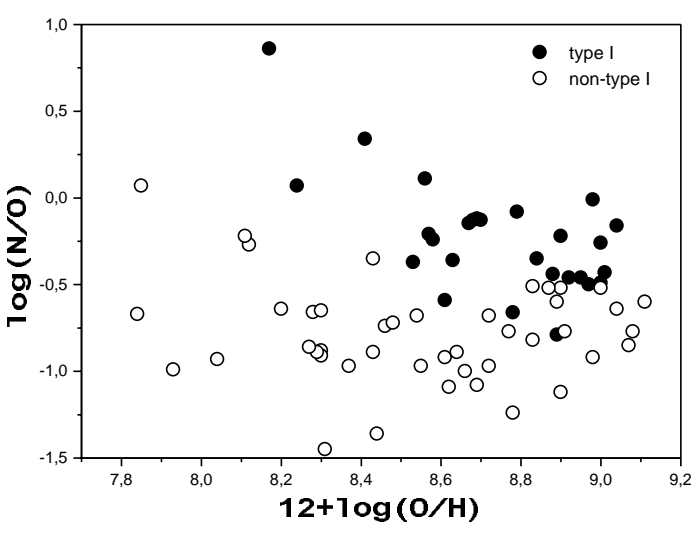

Fig. 1. N/O vs. O/H plot for galactic planetaries where only type I and non-type I objects are discriminated. Data are from Costa \& de Freitas Pacheco (1996)

objects, but the situation is still controversial when nontype I planetaries are considered. Here we revisit this question, using a homogeneous sample constituted of $74 \mathrm{PN}$ (Costa \& de Freitas Pacheco 1996) classified in different groups, according to chemical, morphological, kinematical and spatial criteria (Maciel \& Dutra 1992). This classification, based on the original scheme proposed by Peimbert \& Torres-Peimbert (1983), aggregates planetaries with progenitors of similar mass in the main sequence and, as we shall see, will play a major role in our understanding of the negative correlation between the $\mathrm{N} / \mathrm{O}$ ratio and the oxygen abundance. For the sake of clarity, we recall in Table 5 the main properties of each class, valid only for galactic planetaries. Halo PN, classified as type IV objects, are not included in this table.

To analyse the data, we have first segregated the planetaries of our galactic sample in two main groups, type I and non-type I. Figure 1 shows these objects in the plane $\mathrm{N} / \mathrm{O}$ vs. $\mathrm{O} / \mathrm{H}$. We note that type I objects (filled circles) show a net anti-correlation, but the remaining PN (open circles) display a scatter diagram. In Fig. 2 we have performed the same plot, but now taking into account the class membership. In order to avoid confusion and to strengthen our point, we plotted only type I (filled circles) and type IIb objects. In this plot we see that objects of the same class have a tendency to display a negative correlation, with planetaries having older progenitors being displaced towards lower oxygen abundances for a given $\mathrm{N} / \mathrm{O}$ ratio. Type III PN follow a strip on the left of type IIb, whereas type IIa objects lie on an intermediate position between types I and IIa. A possible interpretation of these plots is that each class is characterized by progenitors within a given mass range (or within a given age interval) and the oxygen variation in each group reflects abundance gradients present in the galactic disk (Maciel \& Köppen 1994). Under these conditions, the observed N/O - $\mathrm{O} / \mathrm{H}$ anti-correlation indicates that the surface enrichment of the progenitor, as a consequence of a dredge-up episode, depends on the metallicity. 
Table 5. Planetary Nebula classes

\begin{tabular}{lcccc}
\hline & Type I & Type IIa & Type IIb & Type III \\
\hline population & young disk & disk & old disk & thick disk (?) \\
initial masses & $2.5-8.0$ & $1.8-2.5$ & $1.2-1.8$ & $0.95-1.2$ \\
$\left\langle Z_{\mathrm{kpc}}\right\rangle$ & 0.15 & 0.28 & 0.42 & 0.66 \\
mean age (Gyr) & 0.3 & 1.4 & 4.3 & 10.2 \\
\hline
\end{tabular}

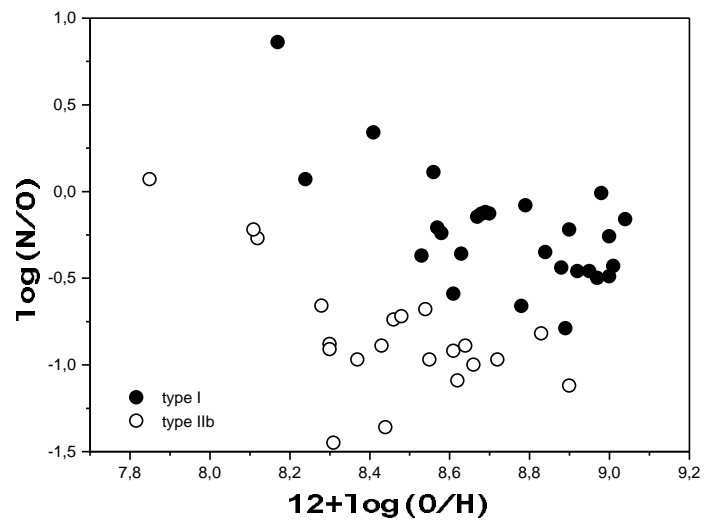

Fig. 2. Same plot as Fig. 1, but for types I and IIb objects only

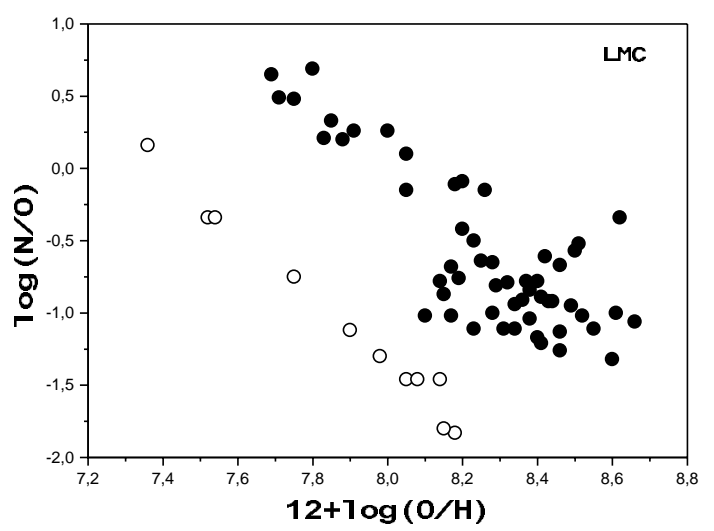

Fig. 3. Same plot for LMC planetaries. Two groups are identified. Filled circles are probably young planetaries, while open circles correspond to objects 5 Gyr old

Guided by these results and using both data by de Freitas Pacheco et al. (1993a,b) and those compiled by Richer (1993), we were able to identify two main PN groups in the LMC. Figure 3 shows the N/O - O/H plot for LMC planetaries, where the different symbols distinguish objects belonging to these different groups. The anti-correlation is clearly detected, in agreement with the conclusions by Henry et al. (1989). The older population has a mean oxygen abundance of $\langle\varepsilon(\mathrm{O})\rangle=7.87$ and, using the evolutionary chemical model for the LMC by de Freitas Pacheco (1998), we found that the mean age of these planetaries is about 5 Gyr. This means that their progenitors have been formed just before the past enhanced star formation episode 3-4 Gyr ago. The second

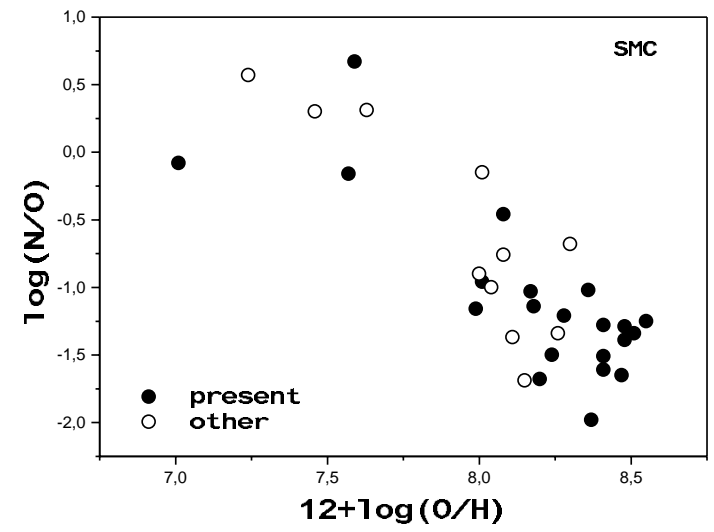

Fig. 4. Same plot for SMC planetary nebulae. Present data are identified as filled circles

group is younger and should be a consequence of the star formation event extending over most of the past 3-4 Gyr, detected on color-magnitude diagrams (Westerlund et al. 1995; Vallenari et al. 1996; Gallagher et al. 1996; Ardeberg et al. 1997).

In Fig. 4 we plotted SMC data. Filled circles correspond to present data, while open circles correspond to literature data compiled by Richer (1993), excluding common objects. Again, the $\mathrm{N} / \mathrm{O}-\mathrm{O} / \mathrm{H}$ anti-correlation is seen but we were not able to discriminate different age groups, in spite of the hint suggested by the observed dispersion of data. The bulk of the objects have abundances in the range $8.0<\varepsilon(\mathrm{O})<8.5$ indicating ages less than 3 Gyr, according to our chemical models for the SMC, to be reported elsewhere. This is consistent with the fact that SMC planetaries have global mean abundances compatible with HII region values, as we have mentioned before. The only exception is SMP25. The rather high electron temperature, in agreement with the value found by Leisy \& Dennefeld (1996), is a signature of a metal-poor nebula, consistent with our abundance determination. Our chemical evolutionary models suggest an age of about $12 \mathrm{Gyr}$ for this object.

In the galactic case, the oxygen spread within a given class may be explained by an age spread as well as by abundance gradients existent in the disk. Probably this is also true for the LMC, since HII region data are consistent with a small gradient (Kolbulnick 1998). It is worth mentioning that such a gradient is not seen in F-supergiant stars (Hill et al. 1995). In the SMC case, no gradients 
were detected (Kolbulnick 1998) and abundance variations must be related to an age effect and/or to incomplete mixing.

\section{Conclusions}

We have performed new spectroscopic observations for a sample of $23 \mathrm{PN}$ in the SMC and derived chemical abundances for $\mathrm{He}, \mathrm{N}, \mathrm{O}, \mathrm{Ne}, \mathrm{S}$ and Ar. Mean abundances agree with values observed in HII regions, suggesting that the bulk of these objects is young, with ages below 3 Gyr. Chemical evolutionary models indicate an enrichment of about 0.20 dex only, in the past 5 Gyr. This is comparable to the rmsd derived when computing sample averages.

We confirm that galactic and Magellanic PN show an anti-correlation between the $\mathrm{N} / \mathrm{O}$ ratio and the oxygen abundance. Such a trend is more evident when planetaries are classified according to the mass range of the progenitors. This "grouping" effect is clearly seen in galactic and in LMC objects. It should also be present in SMC planetaries, but the present sample is not complete, since older $\mathrm{PN}$ are probably missing.

If we discard oxygen conversion into nitrogen for the reasons already mentioned, then the most plausible explanation for such a negative correlation is that besides the mass of the progenitor, the metallicity must affect the efficiency of the dredge-up. Integrated yield contributions from low- and intermediate-mass stars, according to Renzini \& Voli (1981), indicate that the amount of fresh ${ }^{4} \mathrm{He}$ is almost constant with metallicity, whereas a small positive trend is derived for ${ }^{14} \mathrm{~N}$. More recent calculations by Marigo (1999) suggest an opposite behavior: both ${ }^{4} \mathrm{He}$ and ${ }^{14} \mathrm{~N}$ show decreasing integrated yields as the metallicity increases, in agreement with our findings. In the case of ${ }^{4} \mathrm{He}$, Marigo (1999) obtains a higher efficiency not only for decreasing metallicities, but also for increasing stellar masses. The ${ }^{14} \mathrm{~N}$ behavior is more complex: for a given metallicity, there is a stellar mass limit above which the efficiency increases abruptly. This mass limit decreases with metallicity approximately as $\log M_{\lim } \approx 0.66+0.28$ $\log \left(Z / Z_{\odot}\right)$.

As a consequence of such a complex enrichment scenario, one should be cautious when classifying extragalactic planetaries on the basis of chemical abundances only. Reversing the argument, the observed trend of LMC planetaries in the $\mathrm{N} / \mathrm{O}-\mathrm{O} / \mathrm{H}$ plane allowed the identification of a group of objects, whose progenitors were formed at about 5 Gyr ago, suggesting that such a diagram could be an additional tool to investigate extra-galactic PN.

Acknowledgements. This work was partly supported by the brazilian agencies FAPESP (proc. 90/03803-3) and CNPq (proc. 300.818/93-9). T.P.I. acknowledges FAPESP for the present post-doc grant 97/13083-7 at IAG.

\section{References}

Aller L.H., 1983, in IAU Symp. 103, Planetary Nebulae, Flower D.R. (ed.). Dordrecht-Reidel, p. 1

Ardeberg A., Gustafsson B., Linde P., Nissen P.E., 1997, A\&A 322,13

Cardelli J.A., Clayton G.C., Mathis J.S., 1989, ApJ 345, 245

Clegg R.E.S., 1987, MNRAS 229, 31

Costa R.D.D., de Freitas Pacheco J.A., 1996, in IAU Symp. 180, Planetary Nebulae, Habing H., Lamers H.J.G.L.M. (eds.). Kluwer, p. 217

Costa R.D.D., Chiappini C., Maciel W.J., de Freitas Pacheco J.A., 1996, A\&AS 116, 249

de Freitas Pacheco J.A., 1993, ApJ 403, 673

de Freitas Pacheco J.A., Barbuy B., Costa R.D.D., Idiart T.P., 1993a, A\&A 271, 429

de Freitas Pacheco J.A., Costa R.D.D., Maciel W.J., 1993b, A\&A 279, 567

de Freitas Pacheco J.A., 1998, AJ 116, 1701

Dennefeld M., 1989, in Recent Developments of Magellanic Cloud Research, de Boer K.S., Spite F., Stasinska G. (eds.), p. 107

Gallagher J.S., et al., 1996, ApJ 466, 732

Henry R.B., Liebert J., Boronson T.A., 1989, ApJ 339, 872

Hill V., Andrievsky S., Spite M., 1995, A\&A 293, 347

Hill V., Barbuy B., Spite M., 1997, A\&A 323, 461

Kaler J.B., Shaw R.A., Kwitter K.B., 1990, ApJ 359, 392

Kingsburgh R.L., Barlow M.J., 1994, MNRAS 271, 257

Kolbulnick C., 1998, in Abundance Profiles: Diagnostic Tools for Galaxy History, Friedli D., Edmunds M., Robert C., Drissen L. (eds.), PASP Conf. Ser. 147, 108

Köppen J., Acker A., Stenholm B., 1991, A\&A 248, 197

Leisy P., Dennefeld M., 1996, A\&AS 116, 95

Luck R.E., Lambert D.L., 1985, ApJ 298, 782

Maciel W.J., Dutra C.M., 1992, A\&A 262, 271

Maciel W.J., Köppen J., 1994, A\&A 282, 436

Marigo P., 1999, astro-ph/9912341

Osterbrock D.E., 1989, Astrophysics of Gaseous Nebulae and Active Galactic Nuclei. University Science Books, Santa Cruz

Peimbert M., 1985, RMxAA 10, 125

Peimbert M., Serrano A., 1980, RMxAA 5, 9

Peimbert M., Torres-Peimbert S., 1983, Planetary Nebulae, IAU Symp. 103, Flower D. (ed.). Reidel-Dordrecht, p. 233

Peimbert M., Torres-Peimbert S., 1987, RMxAA 15, 117

Renzini A., Voli M., 1981, A\&A 94, 175

Richer M.G., 1993, ApJ 415, 240

Russel S.C., Dopita M., 1990, ApJS 74, 93

Torres-Peimbert S., 1984, in Stellar Nucleosynthesis, Chiosi C., Renzini A. (eds.). Dordrecht-Reidel, p. 3

Torres-Peimbert S., Peimbert M., 1977, RMxAA 2, 181

Vallenari A., Chiosi C., Bertelli G., Aparicio A., Ortolani S., 1996, A\&A 309, 367

Westerlund B.E., Linde P., Lynga G., 1995, A\&A 298, 39 